TAUP 2385-96

\title{
Thermodynamics and the measure of entanglement
}

\author{
Sandu Popescu* \\ Isaac Newton Institute, Cambridge University, 20 Clarkson Road, Cambridge CB3 OEH, U.K. \\ Daniel Rohrlich ${ }^{\dagger}$ \\ School of Physics and Astronomy, Tel Aviv University, Ramat Aviv 69978 Tel-Aviv, Israel
}

(September 24, 2018)

\begin{abstract}
We point out formal correspondences between thermodynamics and entanglement. By applying them to previous work, we show that entropy of entanglement is the unique measure of entanglement for pure states.

3.65.Bz, 89.70.+c
\end{abstract}

Typeset using REVTEX

${ }^{*}$ E-mail: sp230@newton.cam.ac.uk

$\dagger$ E-mail: daniel@ccsg.tau.ac.il 
Quantum entanglement is a most remarkable effect, and we have yet to develop all the tools we need for studying it. One tool we require is a measure of entanglement [1]. How do we quantify entanglement? There have been many ad hoc proposals for measuring entanglement, but until now, the choice among them has been arbitrary. Among the tools in use for studying entanglement are information theory [2] and various definitions of entropy [3 5]. The use of information theory and entropy to analyze entanglement indicates a close correspondence between entanglement and thermodynamics. In part, the correspondence consists of common definitions, such as von Neumann entropy. In part, the correspondence is formal: a formal principle of thermodynamics may apply, mutatis mutandis, to the study of entanglement. The purpose of this note is to exploit the formal correspondence: we show that principles of thermodynamics, applied to previous work on entanglement [3], single out a unique measure of entanglement for pure states.

When Einstein searched for a universal formal principle from which to derive a new mechanics (namely, special relativity) he took for inspiration a general principle of thermodynamics: The laws of nature are such that it is impossible to construct a perpetuum mobile [6]. This general principle (the second law) enabled Carnot to show that all reversible heat engines operating between given temperatures $T_{1}$ and $T_{2}$ are equally efficient. Consider two reversible heat engines; suppose that both absorb heat $Q_{1}$ at $T_{1}$ and expel heat $Q_{2}$ at $T_{2}$, but one does work $W$, and the other does work $W^{\prime}>W$, per cycle. The first engine, if run in reverse, is a refrigerator-absorbs heat $Q_{2}$ at $T_{2}$ and expels heat $Q_{1}$ at $T_{1}$-and requires only work $W$ per cycle. Thus the two engines together could provide $W^{\prime}-W$ in work per cycle without changing their environment. Such a conclusion contradicts the second law, so both engines must do the same work: $W=W^{\prime}$.

The formal correspondence with entanglement is as follows: The laws of nature are such that it is impossible to create (or increase) entanglement between remote quantum systems by local operations [3, 4 . It is clear that quantum mechanics does not allow local operations to create such entanglement, although they may preserve or destroy entanglement [7]. This general principle is the analogue of the second law of thermodynamics. The analogue of a 
reversible heat engine is any reversible transformation, consisting only of local operations, that transforms one entangled state into another. Let two experimenters, Alice and Bob, share pairs of quantum systems in an entangled state. One quantum system in each pair goes to Alice, and the other goes to Bob. In addition, each experimenter may have access to other quantum systems that are not initially entangled. Local operation include any measurements or unitary transformations that Alice performs on her systems, and that Bob performs on his. Alice and Bob can even exchange messages in the usual way (classical messages), but Alice may not send Bob any system entangled with one that she keeps, nor may Bob send such a system to Alice; thus, they cannot create entanglement between their systems [8]. In principle, however, this restriction still allows them to transform entanglement in one pair of systems into entanglement involving other systems. For example, they may, using local operations, transfer entanglement between two spins in a singlet state to two identical spins that initially were uncorrelated, i.e. in a product state, and the original spins will now be in a product state. This transformation is reversible: local operations can transfer the singlet entanglement back to the original spins, leaving the substitute spins uncorrelated.

We may consider more general transformations. Suppose that Alice and Bob share $k$ pairs of systems in an entangled state, and that, by local operations only, they transform the entanglement to $n$ pairs of systems in a different entangled state. Since Bob and Alice have access to other systems that are not initially entangled, $k$ and $n$ may be different. Even if $n>k$, there need be no contradiction with the general principle that it is impossible to create entanglement by local operations, because the state of the $n$ pairs may be less entangled than the state of the original $k$ pairs. If Alice and Bob can transform $k$ pairs in one entangled state into $n$ pairs in another entangled state without destroying any entanglement, then any measure of entanglement must assign the same entanglement to the $k$ initial pairs and the $n$ final pairs. But did they not destroy any entanglement? That is, a question arises with regard to the efficiency of the transformation: could Alice and Bob apply a different set of local operations to obtain the same number $n$ of final pairs from a smaller number $k^{\prime}<k$ of initial pairs? 
The answer is that they cannot, if both transformations are reversible. For if it were possible to transform $k^{\prime}$ of the initial pairs into $n$ of the final pairs by a different transformation, Alice and Bob could then reverse the first transformation and transform the $n$ pairs in the final state to $k$ pairs in the initial entangled state. In doing so, they would have added $k-k^{\prime}$ entangled pairs to their initial supply, contradicting the general principle that it is impossible to create entanglement by local operations [7]. Thus $k^{\prime}=k$.

The reversible local transformations we have assumed are, in fact, consistent with quantum mechanics. Bennett, Bernstein, Popescu and Schumacher [3] have shown that is possible, with local operations only, to transform $k$ systems in an entangled state $\left|\Psi_{A B}^{\prime}\right\rangle$ into $n$ systems in a different entangled state $\left|\Psi_{A B}\right\rangle$. The transformation is reversible when the number of systems becomes arbitrarily large. That is, the ratio $n / k$ tends to a constant in the limit $k \rightarrow \infty$. We can then assign, to $k$ systems in a pure entangled state $\left|\Psi_{A B}\right\rangle$, the same measure of entanglement as (say) $n$ singlet pairs. Thus the problem of defining a measure of entanglement for $k$ pure states reduces to the problem of defining a measure of entanglement for $n$ singlets. At first, it might seem that many such measures, such as $n$, $n^{2}$ and $e^{n}$, would be admissible. But actually, the measure must be proportional to $n$. The reason is that the transformations under consideration are reversible only when the number of systems becomes arbitrarily large [9]. Indeed, the ratio $n / k$ nearly always tends to an irrational number [3], and if the number is irrational, we can never reversibly transform $n$ singlets into a finite number $k$ of systems in the state $\left|\Psi_{A B}\right\rangle$. Reversibility requires us to go to the limit of infinite $n$, and for infinite $n$ there is no way to define total entanglement. We can only define entanglement per system. Here too, thermodynamics provides the formal principle: the thermodynamic limit requires us to define intensive quantities. Likewise, the measure of entanglement must be intensive, i.e. the measure of entanglement of $n$ singlets must be proportional to $n$. It immediately follows that the measure of entanglement for pure states is unique (up to a constant factor). Since the measure of entanglement of $k$ systems $\left|\Psi_{A B}\right\rangle$ approaches the measure of entanglement of $n$ pairs in a singlet state $\left|S_{A B}\right\rangle$, and since the measure is intensive, we have $k E\left(\left|\Psi_{A B}\right\rangle\right)=n E\left(\left|S_{A B}\right\rangle\right)$, where $E$ denotes the measure. 
Thus

$$
E\left(\left|\Psi_{A B}\right\rangle\right)=\lim _{n, k \rightarrow \infty} \frac{n}{k} E\left(\left|S_{A B}\right\rangle\right)
$$

i.e. the entanglement of the state $\left|\Psi_{A B}\right\rangle$ is proportional to the number of singlet pairs per system in the state $\left|\Psi_{A B}\right\rangle$, in this limit. The proportionality constant $\left.\left.E\left(\mid S_{A B}\right)\right\rangle\right)$-measuring the entanglement of a singlet pair - simply defines a conventional unit, and we set it to 1 .

We have shown that the measure of entanglement for pure states $\left|\Psi_{A B}\right\rangle$ is the limit $E\left(\left|\Psi_{A B}\right\rangle\right)$ of Eq. (11). It remains to compute this limit. Indeed, Bennett, Bernstein, Popescu and Schumacher [3] have computed it: $E\left(\left|\Psi_{A B}\right\rangle\right)$ equals the entropy of entanglement of the state $\left|\Psi_{A B}\right\rangle$. The entropy of entanglement is the von Neumann entropy of the partial density matrix seen by either Alice or Bob, and equals the Shannon entropy of the squares of the coefficients of the entangled state in the Schmidt decomposition [3]. The entropy of entanglement is zero for a pair of systems in a product state, and 1 for a pair of spin-1/2 particles in a singlet state; it is never negative. The entropy of entanglement is intensive, as required: if the measure of entanglement of one pair in a state $\left|\Psi_{A B}\right\rangle$ is $E\left(\left|\Psi_{A B}\right\rangle\right)$, then the measure of entanglement of $k$ pairs in the same state is $k \cdot E\left(\left|\Psi_{A B}\right\rangle\right)$. Bennett, Bernstein, Popescu and Schumacher [3] argue that the entropy of entanglement is a good measure of entanglement for pure states, because local operations can interconvert states of equal entropy of entanglement with asymptotically perfect efficiency, but can never increase the entropy of entanglement. We have presented a stronger argument, showing that it is the unique measure of entanglement for pure states.

We arrive at this unique measure by considering all possible local operations on entangled systems. But, in a given setting, the possible local operations may not all be practical. For example, Alice and Bob may not have access to arbitrarily many entangled systems. Then various measures of entanglement that are not valid in principle may be valid (and useful) in practice, simply because Alice and Bob cannot perform the operations that invalidate them. These measures must not increase under the local operations Alice and Bob can perform. A formal statement of this constraint is that if Alice and Bob can, by local operations, trans- 
form an entangled state $\left|\Psi_{A B}\right\rangle$ into entangled states $\left|\Phi_{A B}^{(i)}\right\rangle$ with probabilities $p_{i}$, respectively, then any measure $E$ must satisfy $E\left(\left|\Psi_{A B}\right\rangle\right) \geq \sum_{i} p_{i} E\left(\left|\Phi_{A B}^{(i)}\right\rangle\right)$. This constraint on measures of entanglement is necessary, but not sufficient when all local transformations are allowed.

We have treated pure states. What about density matrices? Is there a unique measure of entanglement for them? Here, too, we can apply thermodynamic principles. Clearly, if there is a reversible transformation between any density matrix and any reference state (such as a singlet), our derivation of the measure of entanglement for pure states extends immediately to density matrices. At present, such reversible transformations are an open question. Two measures of entanglement are particularly natural: the entropy of formation and the entropy of distillation [4]. The former measures the number of singlets Alice and Bob need in order to create a mixed state, while the latter measures the number of singlets that Alice and Bob can extract from a mixed state. If there is a reversible transformation between the entropy of formation and the entropy of distillation, then these measures coincide (as they do for pure states) and define the unique measure of entanglement for mixed states.

\section{ACKNOWLEDGMENTS}

We thank A. Shimony for comments. D. R. acknowledges support from the State of Israel, Ministry of Immigrant Absorption, Center for Absorption in Science (Giladi Program).

\section{NOTE ADDED}

After completing this work, we learned of a paper by V. Vedral, M. B. Plenio, M.A. Rippin and P. L. Knight [10] which assumes, as we do, that local operations cannot create entanglement. Their paper presents a whole class of 'good' entanglement measures. The reason that Vedral et al. do not obtain a unique measure of entanglement, as we do, is that they consider only those local operations that Alice and Bob may perform on each of their entangled pairs in isolation from the other pairs. By contrast, here we consider collective local operations on the pairs, and these imply much stronger constraints on the measure. 


\section{REFERENCES}

[1] See A. Shimony, in The Dilemma of Einstein, Podolsky and Rosen 60 Years Later (Annals of the Israel Physical Society, 12), A. Mann and M. Revzen, eds., Adam Hilger, to appear.

[2] See, e.g., S. L. Braunstein and C. M. Caves, Phys. Rev. Lett. 61, 662 (1988); H. P. Yuen and M. Ozawa, Phys. Rev. Lett. 70, 363 (1993); R. Horodecki, Phys. Lett. A187, 145 (1994); and R. Horodecki and M. Horodecki, Phys. Rev. A54, 1838 (1996).

[3] C.H. Bennett, H. Bernstein, S. Popescu and B. Schumacher, Phys. Rev. A53, 2046 (1996).

[4] C. H. Bennett, G. Brassard, S. Popescu, B. Schumacher, J. A. Smolin and W. K. Wootters, Phys. Rev. Lett. 76, 722 (1996).

[5] C. H. Bennett, D. DiVincenzo, J. Smolin and W. K. Wootters, Phys. Rev. A54, 3824 (1996).

[6] A. Einstein, Autobiographical notes, trans. and ed. by P. A. Schilpp (Chicago: Open Court Pub. Co.) 1979, pp. 50-51.

[7] Quantum mechanics does not allow local operations to increase entanglement, in the following sense: local operations cannot transform any entangled state into the same entangled state plus some new entangled state. To prove that they cannot, it is sufficient to compare the Schmidt decomposition of the initial state with the Schmidt decomposition of the final state (i.e., the direct product of the initial state and the new entangled state). The Schmidt decomposition of the final state has more nonzero terms than the Schmidt decomposition of the initial state. Yet local operations - measurements and unitary transformations - can never increase the number of nonzero terms in the Schmidt decomposition.

[8] By convention, Alice and Bob may exchange classical messages. The logic of this conven- 
tion is as follows. Local operations cannot increase entanglement, but they also cannot increase classical correlations. Thus, if we define entanglement as whatever local operations cannot increase, our definition does not distinguish between entanglement and classical correlations.

[9] We note that quantum mechanics does not allow local operations to increase entanglement even in the limit $n, k \rightarrow \infty$. Suppose initially there are $M$ copies of an entangled state $|\Psi\rangle$, and finally there are $M$ copies of the same state $|\Psi\rangle$ plus $M$ copies of a new entangled state $\left|\Psi^{\prime}\right\rangle$. The scalar product between the initial and final states has norm less than 1 , and in the limit $M \rightarrow \infty$ it vanishes, unless $\left|\Psi^{\prime}\right\rangle$ reduces to a product state. Thus, even in the limit of infinitely many systems, there is no way to produce a final state with more entanglement per system than the initial state.

[10] V. Vedral, M. B. Plenio, M.A. Rippin and P. L. Knight, Phys. Rev. Lett 78, 2275 (1997). 